\title{
Systemic confounders affecting serum measurements of omega- 3 and -6 polyunsaturated fatty acids in patients with retinal disease
}

Anima D. Bühler', Felicitas Bucher', Michael Augustynik', Jan Wöhrl², Gottfried Martin', Günther Schlunck', Hansjürgen Agostini ${ }^{1}$, Daniel Böhringer ${ }^{1}$, Gerhard Pütz ${ }^{2}$ and Andreas Stahl ${ }^{1^{*}}$

\begin{abstract}
Background: Omega-3 polyunsaturated fatty acids (PUFAs) have a highly anti-angiogenic effect in animal models. However, the clinical relevance of omega-3PUFAs in human retinal pathologies remains unclear. The ARED 2 study found no effect of omega-3 PUFA supplementation on progression of age related macular degeneration (AMD). The aim of this study was to compare serum levels of omega-3- and omega-6 PUFAs between patients with diabetic retinopathy (DR), AMD and retinal vein occlusion (RVO), and to identify potential confounders of serum level measurements.

Methods: Venous blood samples were collected from 44 patients with DR, 25 with AMD, 12 with RVO and 27 controls. The lipid phase was extracted and analyzed using mass spectrometry. Retinal disease staging was done by indirect funduscopy and FAG where appropriate. Patient demographics and medical history including current medication and fasting state were acquired. Tukey contrasts for multiple comparisons of the mean and linear regression analysis were used for statistical analysis.

Results: Our data revealed no significant differences in omega-6 PUFA serum levels between patients with AMD, DR, RVO and controls $(p>0.858)$. Uncorrected omega-3 PUFA levels were significantly higher in patients with AMD compared to DR but not compared to controls $(p=0.004)$. However, after correcting for possible confounders such as body mass index (BMI), age, sex, fasting and use of statins, no statistically significant difference remained for serum omega-3 PUFA levels. Fasting was identified as an independent confounder of total omega-6 PUFAs, three individual omega-6 PUFAs and one omega-3 PUFA $(p<0.0427)$. Statin use was identified as an independent confounder of a-linolenic acid (an omega-3PUFA; $p=0.0210$ ).

Conclusion: In this pilot study with relatively low patient numbers, we report significant differences in serum levels of omega-3PUFAs among patients with different types of retinal diseases. However, these differences were not robust for disease specificity after correction for possible confounders in our cohort. Our results demonstrate that serum lipid profiles need to be interpreted with caution since they are significantly altered by variables like fasting and medication use independent from the underlying disease. Correcting for respective confounders is thus necessary to compare serum lipid profiles in clinical studies.
\end{abstract}

Keywords: AMD, DME, RVO, Lipid, Omega-3, PUFA

(Continued on next page)

\footnotetext{
*Correspondence: andreas.stahl@uniklinik-freiburg.de

${ }^{1}$ Eye Center, Medical Center, Faculty of Medicine, University of Freiburg,

Killianstrasse 5, 79106 Freiburg, Germany

Full list of author information is available at the end of the article
} 
(Continued from previous page)

Abbreviations: 4-HDHA, 4-hydroxy-docosahexaenoic acid; AA, Arachidonic acid; AMD, Age-related macular degeneration; AREDS, Age-related eye disease study; BMI, Body mass index; BRVO, Branch retinal vein occlusion; CRVO, Central retinal vein occlusion; DHA, Docosahexaenoic acid; DR, Diabetic retinopathy; EPA, Eicosapentaenoic acid; NAT2, Nutritional AMD Treatment Study; OIR, Oxygen-induced retinopathy; PUFA, Polyunsaturated fatty acid; RVO, Retinal vein occlusion

\section{Background}

Angioproliferative retinal diseases such as diabetic retinopathy or the wet type of age related macular degeneration are responsible for visual impairment among many working age individuals and the elderly [1,2] and lipid mediators have been discussed as part of the pathogenetic cascade in these disorders $[3,4]$.

Omega-3-polyunsatureted fatty acids (PUFAs) are major structural lipids of retinal photoreceptor outer segment membranes and have important structural and protective functions in the retina [5]. The anti-inflammatory effects of some of these omega-3PUFAs such as eicosapentaenoic acid (EPA) and docosahexaenoic acid (DHA) were implicated to play a protective role in AMD development $[5,6]$. In addition, it was also previously described that omega-3PUFAs increase the retinal density of macular pigment, which filters blue light, and has local antioxidant and anti-inflammatory activities [7].

In the mouse model of oxygen-induced retinopathy (OIR), omega-3PUFAs were found to have potent antiangiogenic effects on retinal neovascularization [8]. For example, Sapieha et al. showed that the omega-3 PUFA metabolite 4-hydroxy-docosahexaenoic acid (4-HDHA) directly inhibits endothelial cell proliferation and sprouting angiogenesis via a 5-lipoxygenase dependent pathway. It was also shown that this effect was independent of the anti-inflammatory properties of 4-HDHA [8]. In contrast, the results of the age-related eye disease study 2 (AREDS2) [9] indicate that the addition of the omega-3PUFAs DHA and eicosapentaenoic acid (EPA) to the standard AREDS formulation does not reduce the risk of AMD progression.

Different from AREDS2, however, Merle et al.found serum EPA to be associated with a lower risk for neovascular AMD in 290 patients from the Nutritional AMD Treatment 2 Study (NAT2) who had neovascular AMD in one eye and early AMD in the other eye [10]. Merle et al. also observed in a different study with 936 elderly patients that high plasma omega-3 PUFA levels were associated with a reduced risk for late AMD [11]. In contrast, Kabasawa et al. detected no difference in fatty acid profiles of AMD patients, except for a mild association of EPA with all types of AMD investigated [12].

These contradicting results of various AMD studies demonstrate the need for characterization of confounding parameters which might affect lipid measurements in serum samples. This is not only important for AMD research but also for research in other areas of retinal disease where lipid imbalance may play a role such as diabetic retinopathy (DR) and retinal vein occlusion (RVO). To date, significantly less scientific data exist for DR and RVO compared to AMD with regard to the potential effect of lipids on disease development or progression.

The aim of our study was therefore to identify potential confounders which may affect a perceived correlation of omega3 PUFAs with the degree of AMD, DR or RVO. In addition to omega-3 PUFAs, we also investigated omega-6 PUFAs as well as monounsaturated and saturated fatty acids.

\section{Methods}

Venous blood samples were obtained from 81 patients with DR, AMD or RVO. In addition, samples from 27 control patients without any of the above diseases were collected. Patients who had more than one of the mentioned pathologies were excluded. Diabetic patients were sub-classified in five groups: diabetes without DR (5 patients), mild non-proliferative DR (6 patients), moderate non-proliferative DR (14 patients), severe non-proliferative DR (5 patients) and proliferative DR (15 patients). The grading was done according to the EDTRS-guidelines [13]. Patients with AMD were sub-grouped in four different categories: early dry AMD (4 patients), active wet AMD (12 patients), scarred wet AMD (3 patients) and geographic atrophy (GA; 6 patients). Patients with retinal vein occlusion were distinguished as suffering from branch retinal vein occlusion (BRVO, 7 patients), or central retinal vein occlusion (CRVO, 5 patients).

Additional clinical data were acquired by indirect fundoscopy and FAG where appropriate. For all patients additional information was gathered including medical history, current and past medication (including vitamin supplementation), state of fasting and body mass index (BMI).

The study was registered with the Ethics Committee at the University Hospital Freiburg. After obtaining written informed consent, one serum sample per patient was acquired by venous blood draw and stored at $-80{ }^{\circ} \mathrm{C}$ until being used for lipid extraction. Lipid extraction was performed by the Department of Clinical Chemistry at the University of Freiburg as previously described at a 
temperature of $4^{\circ} \mathrm{C}$ in a two-step procedure [14]. $100 \mu \mathrm{l}$ of serum sample were mixed with $100 \mu \mathrm{l}$ methylpentadecanoate $5 \mathrm{mg} / \mathrm{l}$ as internal lipid standard mixture (analytical standard grade, Sigma, Taufkirchen, Germany). Samples were extracted with $990 \mu \mathrm{l}$ chloroform/methanol (17:1, V/V) for $120 \mathrm{~min}$. The lower organic 17:1 lipid phase extract was collected. The remaining aqueous sample material was re-extracted with $990 \mu \mathrm{l}$ chloroform/ methanol (2:1, V/V) for 120 min. Again, the lower organic 2:1 lipid phase extract was collected. Combined organic phases were then evaporated in vacuum. The dried lipid extracts were dissolved in $70 \mu \mathrm{l}$ chloroform and $30 \mu \mathrm{l}$ of trimethylsulfonium hydroxid (0.25 M in methanol, Sigma Aldrich) were added. To yield respective fatty acid methyl esters, the mixture was stirred for $30 \mathrm{~min}$ at room temperature [15]. Fatty acid methyl esters were analyzed by GC-MS on an Agilent GC 6890/MS 5973 platform (Agilent Technologies Deutschland $\mathrm{GmbH}$, Böblingen Germany). Helium (5.0) was used as carrier gas, injection volume was $1 \mu \mathrm{l}$ and flowrate was $0.4 \mathrm{ml} / \mathrm{min}$ on a SP2380 capillary column (Sigma). Initial temperature was $100{ }^{\circ} \mathrm{C}$, rising with $6{ }^{\circ} \mathrm{C} / \mathrm{min}$ to a final temperature of $220^{\circ} \mathrm{C}$, which was kept for $10 \mathrm{~min}$. Mass spectra were recorded in fullscan mode from $\mathrm{m} / \mathrm{z} 45$ to 800 . Total ion chromatograms (TICs) and fragmentation patterns were acquired by G1701DA GC/MSD Chemstation software. Fatty acid methyl esters were identified by retention time and characteristic mass-to-charge ratios compared to a standard mass chromatogram in the NIST (National Institute of Standards and Technology) mass spectral library. Peak areas were quantitated manually for each identified fatty acid methyl ester. Results for respective fatty acids are presented as percentage of total fatty acid content per sample. Statistical analysis was performed using $\mathrm{R}$ with the following packages: plyr, reshape, car, Hmisc, ggplot2 and multcomp. We applied ANOVA, Tukey contrasts for between-group comparisons and linear regression for inference analyses.

\section{Results}

Our initial analysis of native sample readings without correction for potential confounders suggested significantly higher omega-3PUFA levels in patients with AMD compared to patients with DR $(p=0.00405$; Table 1a). For omega-6 PUFA levels, there was no significant difference between patients with AMD, DR, RVO and controls ( $p>0,858$; Table $1 \mathrm{~b})$.

When analyzing not only the broad disease categories AMD, DR and RVO, but also disease subgroups, there were no significant differences for omega-3 or omega- 6 PUFAs. Two exemplary comparisons across disease subgroups are shown in Fig. 1 for DHA, a major omega-3 fatty acid and arachidonic acid (AA), a major omega-6 fatty acid. Similar patterns without apparent differences
Table 1 Serum omega-3 (A) and omega-6 (B) PUFA levels in patients with different retinal diseases

\begin{tabular}{lllll}
\hline & Estimate & std. error & t value & $\operatorname{Pr}(>|t|)$ \\
\hline A. omega-3 PUFAs & & & & \\
AMD vs Control & 0.48 & 0.25 & 1.92 & 0.222 \\
DR vs Control & -0.28 & 0.22 & -1.26 & 0.585 \\
RVO vs Control & -0.13 & 0.31 & -0.41 & 0.976 \\
DR vs AMD & -0.76 & 0.22 & -3.47 & $0.004^{* *}$ \\
RVO vs AMD & -0.61 & 0.31 & -1.98 & 0.199 \\
RVO vs DR & 0.15 & 0.29 & 0.53 & 0.950 \\
B. omega-6 PUFAs & & & & \\
AMD vs Control & 0.59 & 1.60 & 0.37 & 0.983 \\
DR vs Control & 1.11 & 1.42 & 0.79 & 0.858 \\
RVO vs Control & 0.64 & 2.02 & 0.32 & 0.988 \\
DR vs AMD & 0.52 & 1.44 & 0.37 & 0.983 \\
RVO vs AMD & 0.06 & 2.03 & 0.03 & 1.000 \\
RVO vs DR & -0.47 & 1.90 & -0.25 & 0.994 \\
\hline
\end{tabular}

The table displays group comparisons without correction for potential confounders. $p$-values are shown in the right column and were calculated using ANOVA with Tukey contrasts correcting for multiple testing **signifies $p$-values $<0.01$

across disease subgroups were observed for other individual fatty acids.

Since the primary objective of this study was to determine whether independent variables may act as confounders of PUFA serum measurements, we analyzed our data set using a linear regression model correcting for patient age and sex, body mass index (BMI), the use of oral statin medication and the state of fasting (defined as at least six hours fasting prior to blood sampling). The results shown in Table 2 demonstrate that fasting state is an independent confounder for omega-6, but not omega-3 PUFA measurements. More importantly, the data also demonstrate that none of the three disease entities (DR, RVO, AMD) acts as an independent variable on total omega-3 or omega- 6 PUFA levels in our cohort when using a linear regression model. A full list of fatty acids analyzed can be found as Additional file 1.

Next, we investigated whether individual fatty acids were significantly affected by individual patient variables. Table 3 demonstrates that the fasting state of a patient acts as an independent confounder of total omega-6PUFAs, three individual omega-6 PUFAs and one omega-3 PUFA $(p<0.0427)$. Statin medication was identified as an independent confounder of $\alpha$-linolenic acid (an omega-3 PUFA; $p=0.0210$ ). Interestingly, patient age and BMI were no independent confounders of serum lipid measurements. The small patient number especially in the RVO subgroup has to be considered when interpreting the results of this pilot study. 


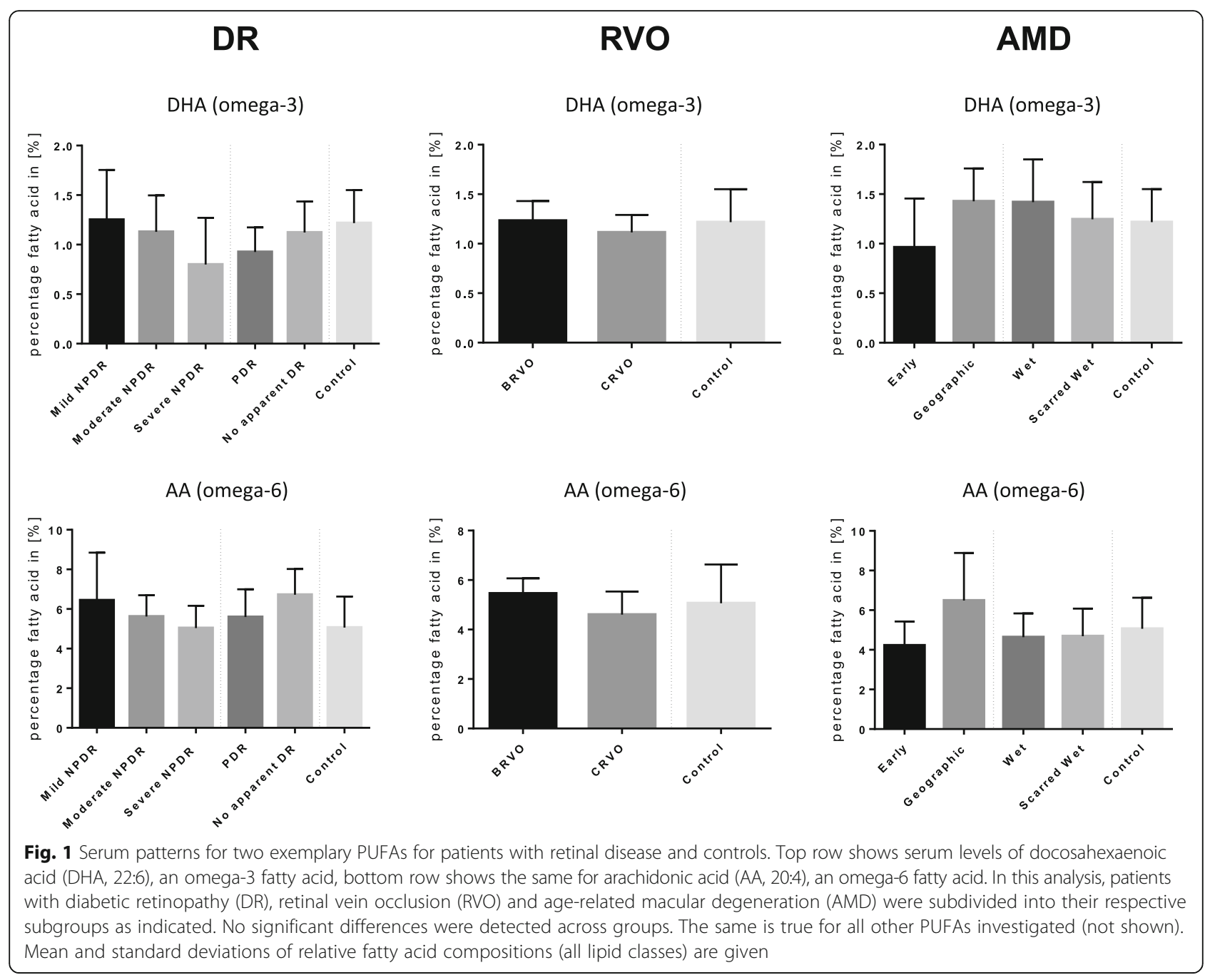

\section{Discussion}

Numerous clinical studies have investigated a possible protective effect of omega-3PUFA supplementation on the progression of AMD. These studies yielded variable and contradicting results $[9,10,16,17]$. The AREDS2 clinical trial, for example, did not find a beneficial effect for omega-3 PUFA supplementation on disease progression [9]. Other studies, in contrast, observed beneficial associations of certain omega-3 PUFA subtypes, such as EPA with AMD risk [10]. For DR and RVO there is significantly less literature compared to AMD regarding a potential association of omega-3 PUFAs with disease stage or progression. In this study, we describe important confounders that may contribute to contradicting results in studies correlating serum lipid levels and eye disease. In addition, our results add valuable data on the less studied disease entities DR and RVO with regard to systemic lipid profiles.

Despite the small sample size of this pilot study, our data clearly suggest that serum lipid levels cannot be used for clinical ophthalmological studies without vigorous correction for potential confounders. The most important confounder is patient fasting state, which affects various unsaturated and saturated fatty acid levels. Most of the existing AMD studies have corrected for potential confounders and report fasting status in their publications. For example, the study by Merle et al. which identified EPA as a risk variable for neovascular AMD did correct for a list of potential confounders including fasting state of the patient [10]. It has to be kept in mind when interpreting lipid studies in general, that not only fasting state but dietary habits in general differ between populations and can potentially impact study results. A meta-analysis published in 2008 , for example, postulates reduced risk for AMD in patients with high dietary intake of Omega-3 PUFAs [18]. For a Japanese AMD population, in contrast, Kabasawa et al. reported no associations with serum omega-3 and omega-6 PUFA levels [12]. Since fish intake in Japan is generally among the highest in the world $[19,20]$, the effect of 
Table 2 Serum omega-3 (A) and omega-6 (B) PUFA levels corrected for potential confounders

\begin{tabular}{lllll}
\hline & Estimate & std. error & $t$ value & $\operatorname{Pr}(>|t|)$ \\
\hline $\begin{array}{lllll}\text { A. omega-3 PUFAs } \\
\text { BMI }\end{array}$ & 0.01 & 0.02 & 0.38 & 0.706 \\
Statin use & 0.03 & 0.25 & 0.12 & 0.905 \\
Fasting & -0.37 & 0.29 & -1.29 & 0.201 \\
Age & 0.01 & 0.01 & 0.55 & 0.582 \\
Sex & -0.10 & 0.21 & -0.48 & 0.635 \\
AMD & 0.45 & 0.31 & 1.43 & 0.155 \\
DR & -0.35 & 0.27 & -1.29 & 0.199 \\
RVO & -0.23 & 0.35 & -0.65 & 0.516 \\
B. omega-6 PUFAs & & & & \\
BMI & -0.19 & 0.12 & -1.55 & 0.125 \\
Statin use & -2.16 & 1.39 & -1.56 & 0.123 \\
Fasting & -5.67 & 1.63 & -3.49 & $0.001^{* * *}$ \\
Age & 0.08 & 0.04 & 1.90 & 0.061 \\
Sex & 0.79 & 1.15 & 0.68 & 0.496 \\
AMD & -1.82 & 1.75 & -1.04 & 0.300 \\
DR & 0.37 & 1.45 & 0.26 & 0.798 \\
RVO & -2.22 & 1.97 & -1.19 & 0.262 \\
\hline Wh & & & & \\
\hline
\end{tabular}

When serum lipid measurements were corrected for the possible confounders body mass index (BMI), statin use, fasting, age and sex of the patient, none of the retinal disease categories acted as an independent variable affecting serum lipid levels. Fasting, in contrast, was identified as an independent variable that significantly affects serum omega- 6 PUFA measurements. ${ }^{* * *}$ signifies $p$-values $\leq 0.001$

omega-3 PUFAs on AMD could potentially have been masked in this population. In addition, changes in serum fatty acid profiles do not necessarily translate into changes in retinal fatty acid profiles.

The second important finding of our study is that, in our cohort, total omega-3 or omega-6 PUFA levels were not independently associated with the presence of AMD.

Table 3 Independent patient variables affecting fatty acid measurements

\begin{tabular}{ll}
\hline Variable & Fatty acid affected by independent variable \\
\hline BMI & - \\
Statin use & $18: 3($ ' $\Omega-3-P U F A)$ \\
Fasting & $14: 00,18: 2($ (' $\Omega-6-P U F A), ~ a 18: 3(' \Omega-3-P U F A)$, \\
& $20: 2(' \Omega-6-P U F A), 20: 4$ (' $\Omega-6-P U F A)$, \\
& total ' $\Omega-6-P U F A s$ \\
Age & - \\
Sex & $16: 00$
\end{tabular}

Multiple regression analysis for individual fatty acids identified several fatty acids whose serum levels were significantly associated with differences in a patient's baseline variables. Fasting state was the most signficiant variable, independently affecting various saturated and unsaturated fatty acid measurements. Statin use and patient sex each affected one of the fatty acids investigated. Patient age and body mass index (BMI) were no independent variables of serum fatty acid measurements in our cohort. Affected fatty acids in this table are noted according to the carboxyl reference system
Different from our study, Merle et al. found associations between AMD and EPA, an omega-3 PUFA [10]. While Merle et al. only investigated patients with neovascular AMD, our study comprised samples from patients with all forms and stages of AMD. The subset of neovascular AMD patients might thus have been too small to pick up potential differences in AMD levels. In contrast to AMD, there is significantly less data available correlating omega-3 and omega-6 PUFA serum levels with the degree of DR or RVO. With regard to RVO, this study is to our knowledge the first to investigate possible associations with serum lipid profiles. In our relatively small patient cohort, we did not find associations of omega-3 or -6 PUFA levels and the presence of RVO. Similarly, DR was not associated with omega- 3 or -6 PUFA levels in our cohort. This is different from saturated fatty acids, for which a positive association with the presence and severity of DR was described [21].

We would like to point out that larger patient numbers would be necessary in order to more reliably investigate clinically relevant differences between patient groups. The main purpose of this manuscript, however, was to identify and flag potent confounders of lipid serum measurement that have to be accounted for when performing these studies. Even with our low patient numbers we were able to identify such confounders - an indication of their potency in affecting results from serum lipid measurements. In this respect, our current manuscript has to be viewed as preparatory work for larger studies by providing important information on methodological aspects of serum lipid measurements that have to be accounted for in larger clinical trials.

\section{Conclusions}

In summary, our data together with the discussed literature emphasize the need for (i) correction for identified confounders of serum lipid profile measurements and (ii) interpretation of lipid profiles in light not only of the disease entity studied but also the underlying patient population. One limitation of our study is the relatively low sample number. Larger patient numbers are thus needed to obtain a full understanding of all factors influencing serum lipid measurements.

\section{Additional file}

Additional file 1: This file contains a list of all fatty acids analyzed for this study. (DOC $41 \mathrm{~kb}$ )

Acknowledgments

The authors thank Johannes Baumann for excellent technical support. 


\section{Funding}

AS is supported by the DFG (STA 1102/5-1) and the German Ophthalmic Society (DOG). This study was supported by a research grant from Novartis Germany.

\section{Availability of data and materials}

The datasets supporting the conclusions of this article are available upon request from the authors. In addition, the results from the linear regression analysis for all fatty acids can be found as supplementary file to this article.

\section{Authors' contributions}

ADB conception and design, acquisition of data, analysis and interpretation of data, drafting the manuscript, revising the manuscript, given final approval and agreed to be accountable. FB, conception and design, acquisition of data, analysis and interpretation of data, revising the manuscript, given final approval and agreed to be accountable. MA, analysis and interpretation of data, revising the manuscript, given final approval and agreed to be accountable. JW, acquisition of data, analysis and interpretation of data, revising the manuscript, given final approval and agreed to be accountable. GM, analysis and interpretation of data, revising the manuscript, given final approval and agreed to be accountable. GS, analysis and interpretation of data, revising the manuscript, given final approval and agreed to be accountable. $\mathrm{HA}$, analysis and interpretation of data, revising the manuscript, given final approval and agreed to be accountable. DB, analysis and interpretation of data, revising the manuscript, given final approval and agreed to be accountable. GP, acquisition of data, analysis and interpretation of data, revising the manuscript, given final approval and agreed to be accountable. AS, conception and design, acquisition of data, analysis and interpretation of data, drafting the manuscript, revising the manuscript, given final approval and agreed to be accountable.

\section{Competing interests}

The funding organizations had no role in study design, data collection and analysis, decision to publish, or preparation of the manuscript. The authors declare that they have no competing interests.

\section{Consent to publish}

Not applicable.

\section{Ethics approval and consent to participate}

The study was registered with the local Ethics Committee at the University Hospital Freiburg. Written informed consent was obtained from all patients.

\section{Author details}

${ }^{1}$ Eye Center, Medical Center, Faculty of Medicine, University of Freiburg, Killianstrasse 5, 79106 Freiburg, Germany. ${ }^{2}$ Institute of Clinical Chemistry and Laboratory Medicine, University Freiburg Medical Center, Freiburg, Germany.

Received: 14 March 2016 Accepted: 23 August 2016

Published online: 05 September 2016

\section{References}

1. Yau JWY, Rogers SL, Kawasaki R, Lamoureux EL, Kowalski JW, Bek T, et al. Global prevalence and major risk factors of diabetic retinopathy. Diabetes Care. 2012;35:556-64.

2. Lim LS, Mitchell P, Seddon JM, Holz FG, Wong TY. Age-related macular degeneration. Lancet Lond Engl. 2012;379:1728-38.

3. Neuringer M, Connor WE, Van Petten C, Barstad L. Dietary omega-3 fatty acid deficiency and visual loss in infant rhesus monkeys. J Clin Invest. 1984;73:272-6.

4. Jeffrey BG, Weisinger HS, Neuringer M, Mitchell DC. The role of docosahexaenoic acid in retinal function. Lipids. 2001;36:859-71.

5. SanGiovanni JP, Chew EY. The role of omega-3 long-chain polyunsaturated fatty acids in health and disease of the retina. Prog Retin Eye Res. 2005;24:87-138.

6. Bazan NG. Neuroprotectin D1-mediated anti-inflammatory and survival signaling in stroke, retinal degenerations, and Alzheimer's disease. J Lipid Res. 2009:50(Suppl):S400-5.

7. Delyfer M-N, Buaud B, Korobelnik J-F, Rougier M-B, Schalch W, Etheve S, et al. Association of macular pigment density with plasma $\omega-3$ fatty acids: the PIMAVOSA study. Invest Ophthalmol Vis Sci. 2012;53:1204-10.
8. Sapieha P, Stahl A, Chen J, Seaward MR, Willett KL, Krah NM, et al. 5Lipoxygenase metabolite 4-HDHA is a mediator of the antiangiogenic effect of $\omega-3$ polyunsaturated fatty acids. Sci Transl Med. 2011;3:69ra12.

9. Age-Related Eye Disease Study 2 Research Group. Lutein + zeaxanthin and omega-3 fatty acids for age-related macular degeneration: the age-related eye disease study 2 (AREDS2) randomized clinical trial. JAMA. 2013;309:2005-15.

10. Merle BMJ, Benlian P, Puche N, Bassols A, Delcourt C, Souied EH, et al. Circulating omega-3 fatty acids and neovascular age-related macular degeneration. Invest Ophthalmol Vis Sci. 2014;55:2010-9.

11. Merle BMJ, Delyfer M-N, Korobelnik J-F, Rougier M-B, Malet F, Féart C, et al. High concentrations of plasma $\mathrm{n} 3$ fatty acids are associated with decreased risk for late age-related macular degeneration. J Nutr. 2013;143:505-11.

12. Kabasawa S, Mori K, Horie-Inoue K, Gehlbach PL, Inoue S, Awata T, et al. Associations of cigarette smoking but not serum fatty acids with agerelated macular degeneration in a Japanese population. Ophthalmology. 2011;118:1082-8.

13. Grading diabetic retinopathy from stereoscopic color fundus photographs-an extension of the modified Airlie House classification. ETDRS report number 10. Early Treatment Diabetic Retinopathy Study Research Group. Ophthalmology. 1991;98:786-806.

14. Ejsing CS, Sampaio JL, Surendranath V, Duchoslav E, Ekroos K, Klemm RW, et al. Global analysis of the yeast lipidome by quantitative shotgun mass spectrometry. Proc Natl Acad Sci U S A. 2009;106:2136-41.

15. Butte WJ. Rapid method for the determination of fatty acid profiles from fats and oils using trimethylsulphonium hydroxide for transesterification. J Chromatogr. 1983;A 261:142-5.

16. Lawrenson JG, Evans JR. Omega 3 fatty acids for preventing or slowing the progression of age-related macular degeneration. Cochrane Database Syst Rev. 2015;4:CD010015.

17. Pinazo-Durán MD, Gómez-Ulla F, Arias L, Araiz J, Casaroli-Marano R, GallegoPinazo R, et al. Do nutritional supplements have a role in age macular degeneration prevention? J Ophthalmol. 2014;2014:901686.

18. Chong EW-T, Kreis AJ, Wong TY, Simpson JA, Guymer RH. Dietary omega-3 fatty acid and fish intake in the primary prevention of age-related macular degeneration: a systematic review and meta-analysis. Arch Ophthalmol. 2008;126:826-33.

19. Sekikawa A, Ueshima H, Kadowaki T, El-Saed A, Okamura T, Takamiya T, et al. Less subclinical atherosclerosis in Japanese men in Japan than in white men in the United States in the post-world war II birth cohort. Am J Epidemiol. 2007;165:617-24.

20. Zhang J, Sasaki S, Amano K, Kesteloot H. Fish consumption and mortality from all causes, ischemic heart disease, and stroke: an ecological study. Prev Med. 1999:28:520-9.

21. Sasaki M, Kawasaki R, Rogers S, Man REK, Itakura K, Xie J, et al. The associations of dietary intake of polyunsaturated fatty acids with diabetic retinopathy in well-controlled diabetes. Invest Ophthalmol Vis Sci. 2015;56:7473-9.

\section{Submit your next manuscript to BioMed Central and we will help you at every step:}

- We accept pre-submission inquiries

- Our selector tool helps you to find the most relevant journal

- We provide round the clock customer support

- Convenient online submission

- Thorough peer review

- Inclusion in PubMed and all major indexing services

- Maximum visibility for your research

Submit your manuscript at www.biomedcentral.com/submit
) Biomed Central 